\title{
Internet Use and Risk Taking Behaviors Among Adolescents
}

\author{
Lakshmana Govindappa • Sekar Kasi • Grace Henry
}

Received: 2 November 2012 / Accepted: 16 May 2013 /Published online: 20 June 2013

(C) Dr. K C Chaudhuri Foundation 2013

To the Editor: With the growing popularity of internet communication applications among adolescents, it has become an important social context for their development. They can utilize the internet either positive way or negative way. The dramatic increase in the use of the internet in recent years has led to pathological use such as internet addiction [1]. The present study, aimed to assess the risk taking behaviors of the adolescents in the internet use. Seventy five samples were drawn from three English medium schools of West Bangalore by using simple random sampling technique and the data was collected using semi structured interview schedule. Results show that $74.7 \%$ of the respondents were male with the mean age of 15.05 $( \pm 1.25) \mathrm{y}$. The mean age at the time of initiation to use the computers was $10.22( \pm 2.49)$ y. Total mean time spent was $7.23( \pm 7.02) \mathrm{h}$ with maximum number of hours spent was $34 \mathrm{~h}$ a week for various purposes that explains the level of dependency on internet use as discussed by various studies $[2,3]$. Majority $(34.7 \%)$ of the respondents had ranked 'playing online games' as their first choice, $29.3 \%$ use internet for academic purpose as their second choice and $29.3 \%$ of respondents ranked the choice of sending emails as third. About $62.7 \%$ play online games, $10.7 \%$ claimed that someone revealed their personal details, $5.3 \%$ reported that they were requested to engage in sexual activities/talk by an adult, $16 \%$ got to know a teenager online and made

L. Govindappa $(\bowtie)$

Department of Social Work, School of Social and Behavioral Sciences, Central University of Karnataka, II Floor, Karya Soudha, Gulbarga University, Gulbarga 585106, Karnataka, India e-mail: lakshmanagsagar@gmail.com

S. Kasi • G. Henry

Department of Psychiatric Social Work, National Institute of

Mental Health and Neurosciences, Bangalore, India contact with him/her by mail/telephone/in person. Majority $(90.7 \%)$ of them had come across nudity and sexual images, $45.3 \%$ have placed their personal details in websites such as Orkut, Facebook, Myspace, and $6.7 \%$ reported that they sought professional help for their pattern of use. This very well explains the risk factor involved for the adolescents while browsing the internet in the absence of proper guidance $[4,5]$. Though, majority (40\%) of the respondents access the computer at home, $66.7 \%$ had no proper guidance and monitoring in using the internet that would increase the risk taking behavior of the adolescents. The studies also report that alienation positively predicted pathological internet use (PIU) directly and also indirectly mediated by leisure services preference $[1,2]$.

The higher use of internet for any purpose as shown in the results also would increase the risk of being alone and decrease the interaction and interpersonal relationship as rightly pointed out by other studies $[1,5]$. Thus the internet should be used in the right way with proper guidance from the care takers and the relevant use of internet by children solely depends on such important factors.

\section{References}

1. Markey PM, Wells SM. Interpersonal perception in internet chat rooms. J Res Pers. 2002;36:134-46.

2. Subrahmanyam K, Lin G. Adolescents on the net: Internet use and well-being. Adolescence. 2007;42:659-77.

3. Nalwa K, Anand AP. Internet addiction in students: A cause of concern. Cyberpsychol Behav. 2003;6:653-6.

4. Bayraktar F, Gün Z. Incidence and correlates of internet usage among adolescents in North Cyprus. Cyberpsychol Behav. 2007;10:191-7.

5. Roger M, Taylor CB, Cunning D, Jones M, Taylor K. Parental restrictions on adolescent internet use. Pediatrics. 2006;118:1804-5. 